\title{
Modeling and Analysis of Train Rear-End Collision Accidents Based on Stochastic Petri Nets
}

\author{
Chao Wu, Chengxuan Cao, Yahua Sun, and Keping Li \\ State Key Laboratory of Rail Traffic Control and Safety, Beijing Jiaotong University, Beijing 100044, China \\ Correspondence should be addressed to Chengxuan Cao; cxcao@bjtu.edu.cn
}

Received 25 September 2014; Revised 28 December 2014; Accepted 2 February 2015

Academic Editor: Zhiwu Li

Copyright (C) 2015 Chao Wu et al. This is an open access article distributed under the Creative Commons Attribution License, which permits unrestricted use, distribution, and reproduction in any medium, provided the original work is properly cited.

\begin{abstract}
We proposed a model of the train rear-end collision accidents based on stochastic Petri nets (SPN) theory. By isomorphic Markov chain model of the proposed accident model, we provide the quantitative analysis of the train rear-end collision accidents. Fuzzy random method is also applied to analyze the performance of the proposed model. In addition, according to the data extracted from a large amount of historical data of the accident statistics, we present a case analysis and discussion. It showed that the results of the proposed train rear-end accident model based on SPN are reasonable in practical applications and can be used to effectively analyze the accidents and prevent loss, and the results may be a reference to the department of railway safety management.
\end{abstract}

\section{Introduction}

Rail transport is an excellent transport mode in reducing pollution and alleviating the traffic congestion and is also a safe and economical way of transportation for passengers and goods. Nowadays the high-speed railway and urban rail transit have been quickly developed and the intensive departure interval strategy has been widely adopted by the railway operators to cope with the demands for transportation. The departure intervals have been as short as possible to maximize the transportation capacity. However, intensive departure intervals also increase the risk of train rear-end accidents. Facing such high-intensive departure intervals, how to ensure the safety of train operations is undoubtedly a challenge for the department of railway safety management. Recently, Chinese people have a lot of doubts of the safety of the rail transport, especially after the Wenzhou high-speed train rearend catastrophic accident in July 23, 2011, and the Shanghai Metro Line 10 rear-end accident in September 27, 2011. In order to ensure the competitive advantage of rail transport, rail transport operators must improve the level of safety to reduce the occurrence of train accidents. Therefore, modeling and analysis of the train rear-end collision accidents have vital practical significance.
In the literatures, many models of accidents and safety analyses have been proposed, such as data mining (DM), Bayesian network (BN), fault tree analysis (FTA), and Petri nets (PN) [1-5]. Mirabadi and Sharifian [1] analyzed the data from past accidents of the Iranian Railway (RAI) by applying the CRISP-DM reference model and the association rules, to discover and reveal unknown relationships and patterns among the data. De Oña et al. [3] presented BN to describe accidents that involve many interdependent variables. The relationship and structure of the variables can be studied and trained from accident data. It does not need to know any predefined relationships between dependent and independent variables.

By the principles of logical deductive analysis, the fault tree model analyzes accidents from a possible top event and analyzes its causes by layers, until we find out all the background events that lead to the accident. Li [6] carried out the fault tree analysis in which the train rear-end accident is seen as the top event and investigated the patterns of the accident to analyze various events which can cause the accident. He also discussed the security elements and strategies of complex safety-critical system from a macro point of view. 
Although all of the models presented in the literature have been recognized as a powerful tool for accident analysis, the majority is only suitable for describing small static system and cannot analyze the dynamic system and it is difficult to analyze the accident quantitatively [7]. As a tool for discrete events' simulation, PN can avoid these deficiencies [8]. Dynamic changes in the PN induced by transition firing make it possible to analyze the dynamic system. In addition, being isomorphic to the state space of the Markov chain (MC), the PN makes analyzing the accident quantitatively convenient.

The main contributions of this paper are as follows. First, we proposed a model of train rear-end collision accidents based SPN theory instead of conventional method such as fault tree analysis. Second, our model can be used for quantitative analysis of the accidents by being isomorphic to MC. Third, fuzzy random method is applied to analyze the performance of the model, which can improve the reliability of the results of the quantitative analysis. Finally, according to the data extracted from a large amount of historical data of the accident statistics, we have carried out a case analysis and discussion, which may be useful to the department of railway safety management.

The remainder of this paper is organized as follows. Fundamental theory of SPN and theoretical basis of system modeling and performance analysis using SPN are given in Section 2. The model of train rear-end collision accidents based on SPN theory is proposed in Section 3. A case analysis and discussion are presented in Section 4. The last section is the conclusion of this paper.

\section{Methods}

Dr. Petri [9] proposed Petri nets theory in 1962 when he developed information flow model for computer operation system. At present, PN is extensively applied to modeling, performance analysis, and control research for discrete event dynamic system [10]. As a system model, PN is dynamic and concurrent. In PN standard graphic presentation, the sign "O" indicates place and the sign "|" or pane indicates transition [11].

\subsection{Fundamentals of $P N$}

(1) Definition: in general, a PN is defined as follows: $\sum=$ $\left(P, T, F, K, W, M_{0}\right)$, where

(a) $P=\left\{p_{1}, p_{2}, \ldots, p_{n}\right\}, n>0$, refers to the finite set of place;

(b) $T=\left\{t_{1}, t_{2}, \ldots, t_{m}\right\}, m>0$, refers to the finite set of transition;

(c) $F$ is an ordered pair set consisting of $P$ and $T$ and satisfies $F \subseteq P \times T \cup T \times P, P \cap T=\varnothing$, and $T \cup P \neq \varnothing$;

(d) $K$ is a capacity function;

(e) $W$ is a weight function, weighting all arc lines; $w(p, t)$ or $w(t, p)$ is used to denote the weight of directed arc going from $p$ to $t$ or $t$ to $p$; (f) $M$ is the marking reflecting token distribution in all places and $M_{0}$ is the initial marking.

(2) Enabling and stimulation rules of transition are as follows:

(a) transition $t \in T$ is enabled, when and only when, in respect of all $p \in P, w(p, t)=1, M(p)>0$;

(b) after the enabled transition $t$ is stimulated by the marking $M$, a new marking $M^{\prime}$ is generated, in respect of $\forall p \in P$, which is

$$
M^{\prime}(p)= \begin{cases}M(p)-w(p, t), & p \in{ }^{o} t, \\ M(p)+w(p, t), & p \in t^{o}, \\ M(p)+w(p, t)-w(p, t), & p \in{ }^{o} t \wedge p \in t^{o}, \\ M(p), & \text { other. }\end{cases}
$$

2.2. Theoretical Basis of System Modeling and Performance Analysis Using SPN. Description of a system by PN is usually based on two concepts: event and state. PN does not only present the graph of a system, but also provide the mathematical description of the system [12]. To describe the dynamic action of a system, execution of the processing task can be indicated by corresponding transition firing. In the $\mathrm{PN}$, the transferring of tokens expresses the information process. The markings of the PN denote the special state of the system. Forward markings decide the set of all possible states of the system with a given initial state.

PN provides a new description tool for system performance analysis [13]. In the continuous-time SPN, certain time delay is needed for a transition from being generable to practical firing; that is, the period from a transition being generable to its firing is regarded as a continuous random variant which is subject to exponential distribution. When the firing rate of transitions is exponentially distributed and the markings are countable, it has been proved that a continuous-time SPN is isomorphic to a continuous-time MC [14]. So each marking of SPN is mapping into a state of $\mathrm{MC}$ and the occurrence graph of SPN is isomorphic to the state space of MC.

Therefore, referring to the SPN occurrence graph which is isomorphic to a homogeneous $\mathrm{MC}$, we can utilize the theory of random processes to analyze our model. The application of SPN to system performance analysis is usually as follows.

(1) Build the SPN model for the system.

(2) Define the possible states of SPN and derive its reachable marking set.

(3) Obtain the isomorphic MC of SPN.

(4) Analyze the system performance based on the stable probability of MC.

Based on its stable probability, we can further analyze the system performance indexes and time characteristics, analyze the busyness and work efficiency of the whole systems 
or transitions, identify main factors affecting the system performance, and analyze the resources distribution and optimization plans for the system under different command and dispatch methods.

\section{Modeling and Analysis of Train Rear-End Collision Accidents Based on SPN}

\subsection{Modeling of Train Rear-End Collision Accidents Based} on SPN. In this paper, we consider the case of one track only in the one-way and assume that the collision avoidance systems such as signal control system, train distance control system, train state communication and control system, and the danger alarm system are applied in the problem. The train track signal failure may be caused by some environmental factors such as lightning strike. According to $\mathrm{Li}$ [6] and our knowledge and understandings of the railway traffic, the major procedure of a train rear-end collision accident is shown in Figure 1. Based on the major procedure of a train rear-end collision accident and the theory of SPN, we proposed the train rear-end collision accidents model as shown in Figure 2.

Places and transitions in Figure 2 are explained as follows. Finite set of places $P$ are as follows: $P_{1}$ : two successive trains are driving normally; $P_{2}$ : velocity of the follow-up train is greater in two successive trains; $P_{3}$ : velocity of follow-up train is still greater; $P_{4}$ : it refers to risk of train rear-end collision accident; $P_{5}$ : it refers to intervention of automatic train protection system (ATP); $P_{6}$ : ATP fails to intervene; $P_{7}$ : ATP intervenes successfully; $P_{8}$ : it refers to intervention of dispatcher; $P_{9}$ : dispatcher intervenes successfully; $P_{10}$ : it refers to effectiveness judgment of dispatcher's measures; $P_{11}$ : dispatcher fails to intervene; $P_{12}$ : it refers to intervention of driver; $P_{13}$ : driver detects the abnormal; $P_{14}$ : it refers to effectiveness judgment of braking; $P_{15}$ : it refers to train rearend collision accident; $P_{16}$ : trains are safe controlled.

Finite set of transitions $T$ are as follows: $t_{1}$ : it refers to velocity difference in two successive trains (velocity of follow-up train is greater); $t_{2}$ : interval distance in two successive trains is much larger than the minimum instantaneous distance; $t_{3}$ : interval distance in two successive trains is approaching the minimum instantaneous distance; $t_{4}$ : velocity difference is eliminated; $t_{5}$ : interval distance in two successive trains is decreasing; $t_{6}$ : ATP works automatically; $t_{7}$ : ATP fails to work; $t_{8}$ : ATP works normally; $t_{9}$ : dispatcher intervenes; $t_{10}$ : dispatcher does not detect or neglect the abnormal; $t_{11}$ : dispatcher detects the abnormal; $t_{12}$ : dispatcher takes measures; $t_{13}$ : dispatcher's measures fail to work; $t_{14}$ : driver intervenes; $t_{15}$ : ATP adjusts the train's driving state automatically; $t_{16}$ : dispatcher's measures work; $t_{17}$ : driver detects the abnormal; $t_{18}$ : driver does not detect the abnormal; $t_{19}$ : driver operates to brake; $t_{20}$ : train fails to stop before rear-end collision accident happened; $t_{21}$ : driver stops the train successfully; $t_{22}$ : the train's driving state is adjusted; $t_{23}$ : it refers to cleaning up for restoring the line operation. Process. According to the SPN model as shown in Figure 2,

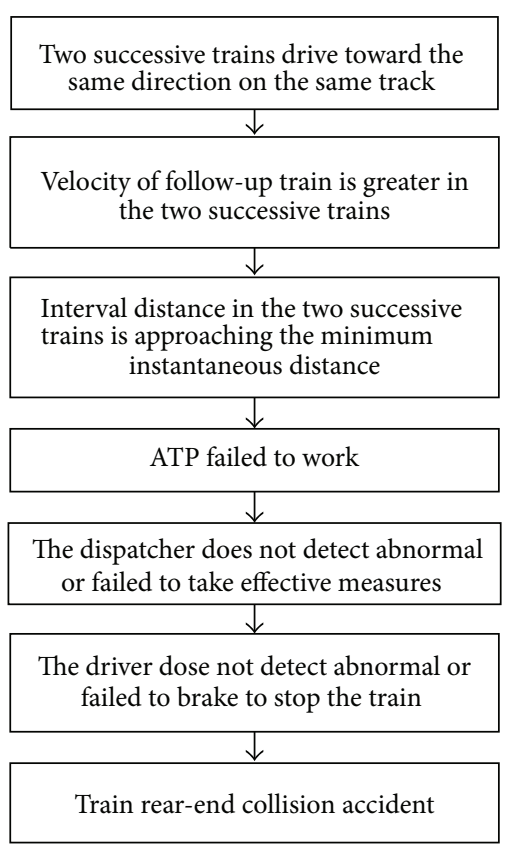

FIGURE 1: Major procedure of occurrence of a train rear-end collision accident.

we can obtain its isomorphic MC in Figure 3, where $M_{1}$ is the initial marking containing only one token in the place $P_{1}$, and the Markov state space can be obtained as $M_{1}$ to $M_{16}$, as a result of the transferring of the token and the firing of different sets of transitions. Since most process service time is close to the exponential distribution and the exponential distribution simplifies the analysis of the random network, we assume that the firing rate of each transition is exponentially distributed in the model. Here, the average firing rate of transitions $t_{1}, t_{2}, \ldots, t_{23}$ are $\lambda_{1}, \lambda_{2}, \ldots, \lambda_{23}$, respectively.

From the MC of the SPN model, the following conclusions can be obtained.

(1) No obstruction occurred in the entire process, and the transform of token is smooth. The process does not wait indefinitely for a state, indicating that all transitions in the process of the train rear-end collision accidents are likely to be implemented in a certain period of time. The successful implementation of each transition is the basis for the next transition to be implemented successfully.

(2) A state $M_{i}$ does not exist, which cannot be reached, namely, no state can never occur, and a deadlock state does not exist in the model.

\section{Case Analysis and Discussion}

4.1. Performance Analysis of SPN Model. Assuming $n$ states located in the MC, we can immediately derive the transfer rate matrix $Q=\left[q_{i, j}\right], 1 \leq i, j \leq n$. The element of the transfer matrix is obtained intuitively as follows. 


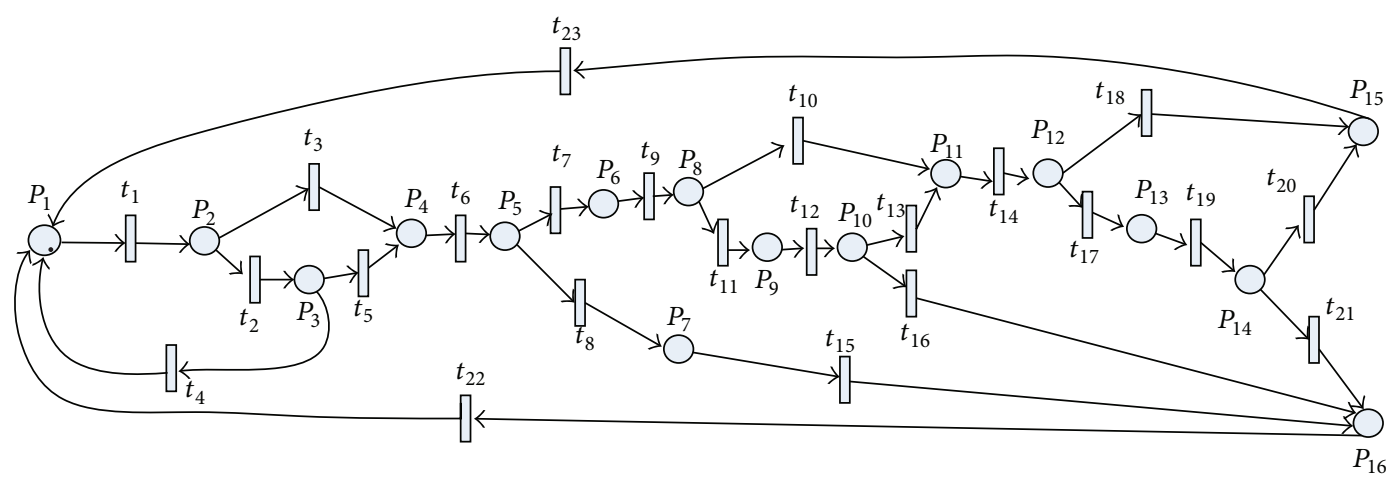

FIGURE 2: Model of a train rear-end collision accident based on stochastic Petri nets.

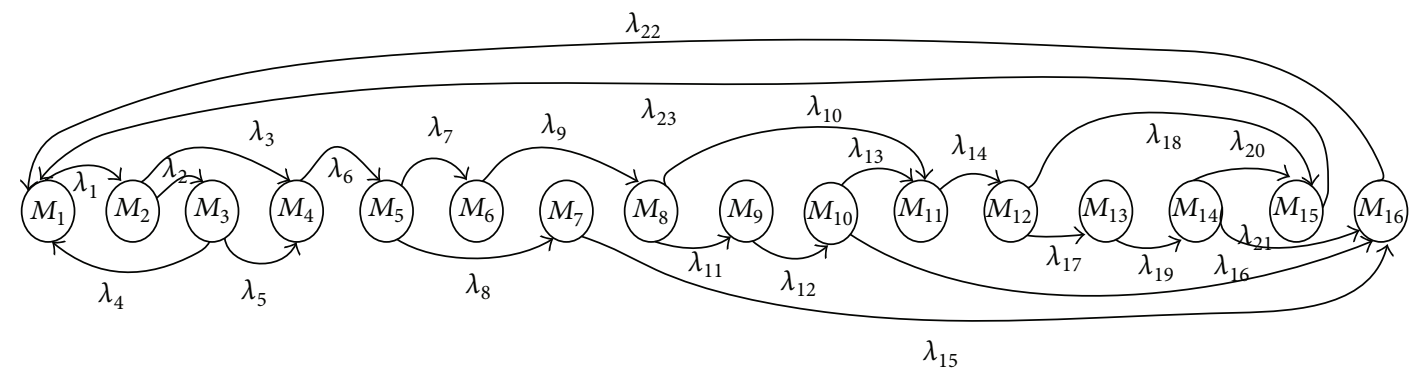

FIgURe 3: Markov chain of the stochastic Petri nets model.

(1) For the element $q_{i, j}$ which is located in nondiagonal, when there is an arc connecting state $M_{i}$ with state $M_{j}$, then the firing rate $\lambda_{i}$ marked on the arc is the value of $q_{i, j}$; if there is not an arc connecting state $M_{i}$ with state $M_{j}$, then $q_{i, j}=0$.

(2) For the element $q_{i, j}$ which is located in diagonal, the negative number of the sum of the rate $\lambda_{i}$ marked on the arcs which are outputting from the state $M_{i}$ is the value of $q_{i, j}[12]$.

Assuming that the stable probability of the $n$ states in the $\mathrm{MC}$ is a row vector $X=\left(x_{1}, x_{2}, \ldots, x_{n}\right)$, we can get the following linear equations according to the Markov process:

$$
\begin{gathered}
X Q=0, \\
\sum_{i} x_{i}=1, \quad 1 \leq i \leq n .
\end{gathered}
$$

Since $\lambda_{i}$ is extracted and estimated on the basis of the statistics data, which is collected from the previous train accidents investigations, there may be incompletion, inaccuracy, and uncertainty. Therefore, using different fuzzy degrees, (2) can be transferred into the fuzzy stable probability equations, and, solving these fuzzy equations, we can calculate the steady state probability. Because the value $\lambda_{i}$ is usually around a certain value $\lambda$, we can use the triangle membership functions to represent fuzzy numbers. Mathematically, the triangle membership function $u_{\lambda_{i}}(x)$ can be expressed as follows [15]:

$$
u_{\lambda_{i}}(x)= \begin{cases}0, & x \leq a_{i 1}, \\ \frac{\left(x-a_{i 1}\right)}{\left(a_{i 2}-a_{i 1}\right)}, & a_{i 1} \leq x \leq a_{i 2}, \\ \frac{\left(a_{i 3}-x\right)}{\left(a_{i 3}-a_{i 2}\right)}, & a_{i 2} \leq x \leq a_{i 3}, \\ 0, & x \geq a_{i 3} .\end{cases}
$$

The fuzzy variables $\lambda_{i}$ can be represented by a triple $\left(a_{1}, a_{2}, a_{3}\right)$, and the parameter $a_{i 2}$ defines the maximum degree of $\lambda_{i}$; namely, $u_{\lambda_{i}}\left(a_{i 2}\right)=1$; parameters $a_{i 1}$ and $a_{i 3}$ define the minimum limit and the maximum limit of the fuzzy number. In addition, the triangle membership function $\left(a_{1}, a_{2}, a_{3}\right)$ defines a $\alpha$-cut of the fuzzy number. In fact, the $\alpha$-cut $A^{(\alpha)}=\left[a_{1}^{(\alpha)}, a_{3}^{(\alpha)}\right]$ defines a confidence interval of the triangular fuzzy number expressed as $A^{(\alpha)}=\left[a_{1}+\left(a_{2}-\right.\right.$ $\left.\left.a_{1}\right) \alpha, a_{3}-\left(a_{3}-a_{2}\right) \alpha\right]$ (see Figure 4).

Because a concrete source which can provide the values of firing rate $\lambda_{i}$ does not exist, we first obtained the data of the frequency of related events of the responding transition from the statistics data of the previous train accidents investigations, and, according to it, we get the values of the firing rate $\lambda_{i}$ after our proper estimation and modification, which are listed in Table 1 with a unit of bout per hour. Since the uncertainty of the data has been considered by applying the fuzzy random method, the data are representative to be used for the further analysis. 
TABLE 1: Value of the firing rate $\lambda_{i}$.

\begin{tabular}{cccccccccccccc}
\hline$\lambda_{1}$ & $\lambda_{2}$ & $\lambda_{3}$ & $\lambda_{4}$ & $\lambda_{5}$ & $\lambda_{6}$ & $\lambda_{7}$ & $\lambda_{8}$ & $\lambda_{9}$ & $\lambda_{10}$ & $\lambda_{11}$ & $\lambda_{12}$ \\
\hline 10 & 2 & 2 & 4 & 4 & 10 & 1 & 4 & 10 & 1 & 1 \\
\hline$\lambda_{13}$ & $\lambda_{14}$ & $\lambda_{15}$ & $\lambda_{16}$ & $\lambda_{17}$ & $\lambda_{18}$ & $\lambda_{19}$ & $\lambda_{20}$ & $\lambda_{21}$ & $\lambda_{22}$ & $\lambda_{23}$ \\
\hline 1 & 1 & 10 & 4 & 4 & 1 & 10 & 2 & 2 & 10 & 1 \\
\hline
\end{tabular}

TABLE 2: Crisp number and triangular number of the firing rate $\lambda_{i}$.

\begin{tabular}{lccr}
\hline$\lambda_{i}$ & Crisp number & Spread & Triangular fuzzy number \\
\hline$\lambda_{1}, \lambda_{6}, \lambda_{9}, \lambda_{12}, \lambda_{15}, \lambda_{19}, \lambda_{22}$ & 10 & $\pm 20 \%$ & $(8,10,12)$ \\
$\lambda_{4}, \lambda_{5}, \lambda_{8}, \lambda_{11}, \lambda_{16}, \lambda_{17}$ & 4 & $\pm 15 \%$ & $(3.4,4,4.6)$ \\
$\lambda_{2}, \lambda_{3}, \lambda_{20}, \lambda_{21}$ & 2 & $\pm 10 \%$ & $(1.8,2,2.2)$ \\
$\lambda_{7}, \lambda_{10}, \lambda_{13}, \lambda_{14}, \lambda_{18}, \lambda_{23}$ & 1 & $\pm 5 \%$ & $(0.95,1,1.05)$ \\
\hline
\end{tabular}

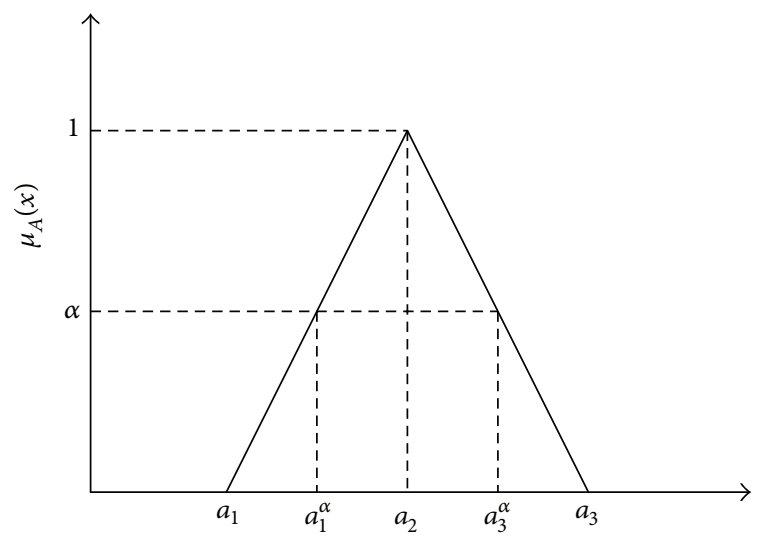

FIGURE 4: The $\alpha$-cut of a triangular fuzzy number.

In order to account for the uncertainties in the data, the obtained crisp data are converted into the fuzzy numbers. More specifically crisp numbers in the extracted data are converted into fuzzy numbers with a known spread. Thus we can get the triangular fuzzy numbers of $\lambda_{i}$ as listed in Table 2 .

Taking $(0.9,1.0,1.1)$ as the triangular fuzzy numbers of the sum of stable probability, we calculate the fuzzy stable probability in the condition of triangular fuzzy number $\lambda_{i}$. Defuzzification is necessary to convert the fuzzy output to a crisp value, as most of the actions or decisions implemented by human or machines are binary or crisp. Out of the existence of the various defuzzification techniques in the literature, center of gravity (COG) method is selected due to its property that it is equivalent to meaning of data [16]. If the membership function $u_{\lambda_{i}}(x)$ of the output fuzzy set $A$ is described on the interval $\left[x_{1}, x_{2}\right]$, then COG defuzzification value $\bar{x}$ can be defined as

$$
\bar{x}=\frac{\int_{x_{1}}^{x_{2}} x \cdot u_{\lambda_{i}}(x) d x}{\int_{x_{1}}^{x_{2}} u_{\lambda_{i}}(x) d x} .
$$

Based on the COG method, the crisp values of the stable probability are calculated, since the place's busy probability refers to the probability of events or states in the train rearend accident process. So we get the results as follows:

$$
\begin{aligned}
& P\left[M\left(p_{1}\right)=1\right]=x_{1}=0.104, \\
& P\left[M\left(p_{2}\right)=1\right]=x_{2}=0.260, \\
& P\left[M\left(p_{3}\right)=1\right]=x_{3}=0.065, \\
& P\left[M\left(p_{4}\right)=1\right]=x_{4}=0.078, \\
& P\left[M\left(p_{5}\right)=1\right]=x_{5}=0.156, \\
& P\left[M\left(p_{6}\right)=1\right]=x_{6}=0.016, \\
& P\left[M\left(p_{7}\right)=1\right]=x_{7}=0.062, \\
& P\left[M\left(p_{8}\right)=1\right]=x_{8}=0.031, \\
& P\left[M\left(p_{9}\right)=1\right]=x_{9}=0.013, \\
& P\left[M\left(p_{10}\right)=1\right]=x_{10}=0.025, \\
& P\left[M\left(p_{11}\right)=1\right]=x_{11}=0.056, \\
& P\left[M\left(p_{12}\right)=1\right]=x_{12}=0.011, \\
& P\left[M\left(p_{13}\right)=1\right]=x_{13}=0.004, \\
& P\left[M\left(p_{14}\right)=1\right]=x_{14}=0.011, \\
& P\left[M\left(p_{15}\right)=1\right]=x_{15}=0.033, \\
& P\left[M\left(p_{16}\right)=1\right]=x_{16}=0.075 .
\end{aligned}
$$

A comparison of the place's busy probability about whether to introduce the fuzzy random method is carried out. In Figure 5, 1, 2, 4, 5, and 6 represent the place's busy probability value in the condition that the firing rate of each transition takes its $a_{1}, a_{1}^{(0.5)}, a_{2}, a_{3}^{(0.5)}$, and $a_{3}$, respectively, as showed in the triangle membership function $\left(a_{1}, a_{2}, a_{3}\right)$; 3 represents each place's busy probability's defuzzification value. We observe that the defuzzification values of place's busy probability are more stable compared to the direct use of crisp firing rate of the transitions, which demonstrate that fuzzy mathematical method accounted for the uncertainties 


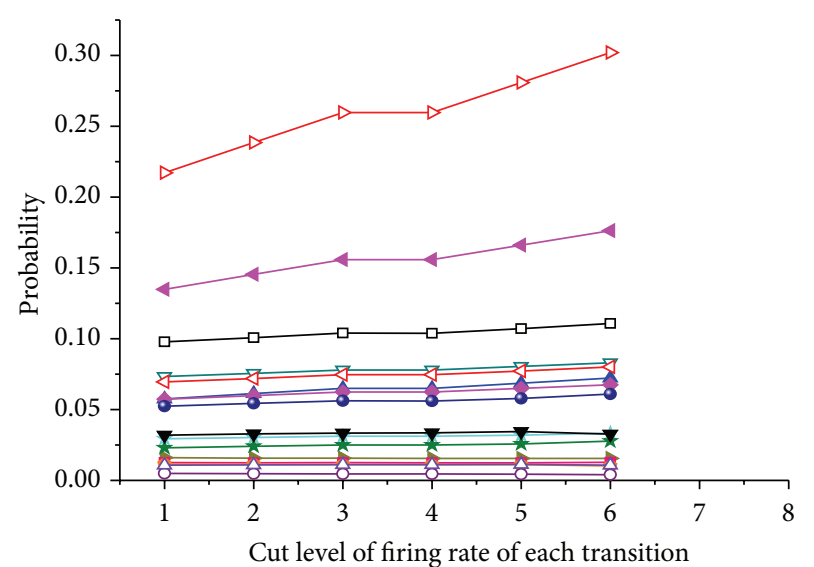

$$
\begin{aligned}
& \because p_{1} \quad \leftarrow p_{5} \quad \rightarrow-p_{9} \quad \multimap p_{13} \\
& \rightarrow p_{2} \quad \rightarrow p_{6} \quad \star p_{10} \quad \backsim p_{14} \\
& \neg p_{3} \quad \neg p_{7} \quad \multimap p_{11} \quad \longrightarrow-p_{15} \\
& \rightarrow-p_{4} \quad \longleftarrow p_{8} \quad \longrightarrow p_{12} \quad \triangleleft p_{16}
\end{aligned}
$$

Figure 5: Stable probability of varying $\alpha$-cut level of firing rate of each transition.

of the firing rate of the transitions. Thus the result is more accurate and reliable.

In addition, from the above data it can be seen that the busy probabilities of places $P_{2}$ and $P_{5}$ are larger relatively. Place $P_{2}$ refers to the state that velocity of the follow-up train is greater in two successive trains. There are many reasons leading to this state, such as the driver violating the driving instruction usually represented in speeding or slowly driving, the automatic control system being abnormal, or the dispatcher's misjudgment. On the one hand, the dispatcher's misjudgment is reflected by the dispatcher's belief that the front train cannot be caught up by the following train in the driving sections to conduct the risk instruction, which suggests that the dispatcher put too much faith in the driver and neglected risk; on the other hand, it is reflected by the fact that the dispatcher may believe in the collision avoidance system which can prevent the accident, which suggests that the dispatcher put too much faith in the technology.

In all of the factors leading to the state $P_{2}$, some cannot be avoided because it is the need of dispatching operation management. In this case, we must be careful and should not put too much faith in the driver or technology to neglect the unexpected adventure and to conduct any risk instruction. Other risk factors can be reduced or eliminated by strengthening the management of drivers and other safety measures.

The large busy probability of place $P_{2}$ inevitably leads to a relatively large busy probability of place $P_{5}$ (intervention of ATP). Because only a very small number of cases of this velocity difference are eliminated naturally, a train generally has to rely on ATP to adjust automatically the train driving status to ensure the safe driving of trains. In case the ATP worked normally in the long-term, train drivers or dispatchers can easily depend on the vital role of the ATP. It is the reason why the accident tends to happen once ATP is not working properly. Therefore, it is more crucial for the

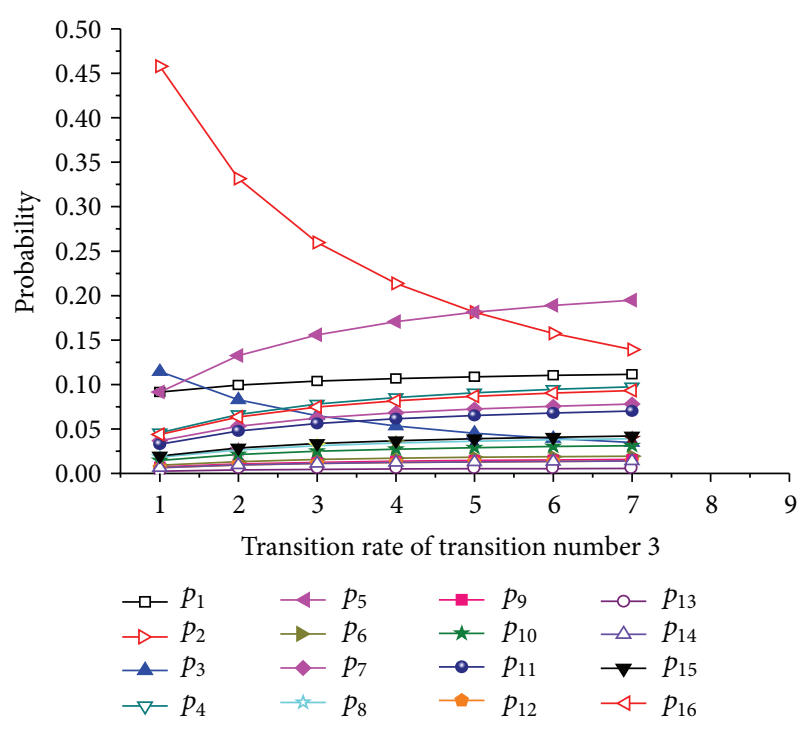

FIgURE 6: Stable probabilities of varying transition rate $\lambda_{3}$.

day-to-day maintenance of railway safeguard equipment and facilities to ensure the safe driving of trains. In addition, the vigilance of the train driver and dispatcher should be strengthened in case of the ATP abnormalities.

Furthermore, the obtained result is consistent with the actual one that the busy probability of place $P_{15}$ (the train rear-end collision accident) is not very large. However, no matter how low the probability is, it does not mean that the accident does not happen. Even if we can accurately estimate the accident, we cannot accurately predict the moment of the accident. Because it involves significant consequences, everyone from the top management to the low level employees should always be vigilant and keep a high level of security alert.

4.2. Discussion. If we change the value of the firing rate $\lambda_{3}$ and values of $\lambda_{1}, \lambda_{2}, \lambda_{4}, \ldots, \lambda_{23}$ remain unchanged, we can get Figure 6, where $1, \ldots, 7$ represent the stable probability value (namely, the place's busy probability) in the condition of the firing rate of transition $t_{3}$ taking $0,0.5 \lambda_{3}, \lambda_{3}, 1.5 \lambda_{3}$, $2 \lambda_{3}, 2.5 \lambda_{3}, 3 \lambda_{3}$, respectively. If we change the value of the firing rate $\lambda_{7}, \lambda_{8}, \lambda_{10}, \lambda_{11}, \lambda_{13}, \lambda_{16}, \lambda_{18}, \lambda_{20}, \lambda_{21}$, we can also get Figures $7-15$ similarly.

In Figure 6 , if $\lambda_{3}$ increases, that is, the frequency of interval distance in two successive trains is approaching to the minimum instantaneous distance increases, the place's busy probability of $P_{4}, P_{5}$, and $P_{15}$ increases remarkably. This shows that the intensive departure interval strategy will significantly increase safety risk and the probability of accidents. Thus we should make a tradeoff between the risk of accidents and intensive departure interval strategy which aim to improve the transport capacity.

From Figures 7 and 8, we find out that ATP plays a very important role as the first barrier for protection of train running security. Once the frequency of ATP fails to work increasingly or the frequency of ATP work normally declines, 


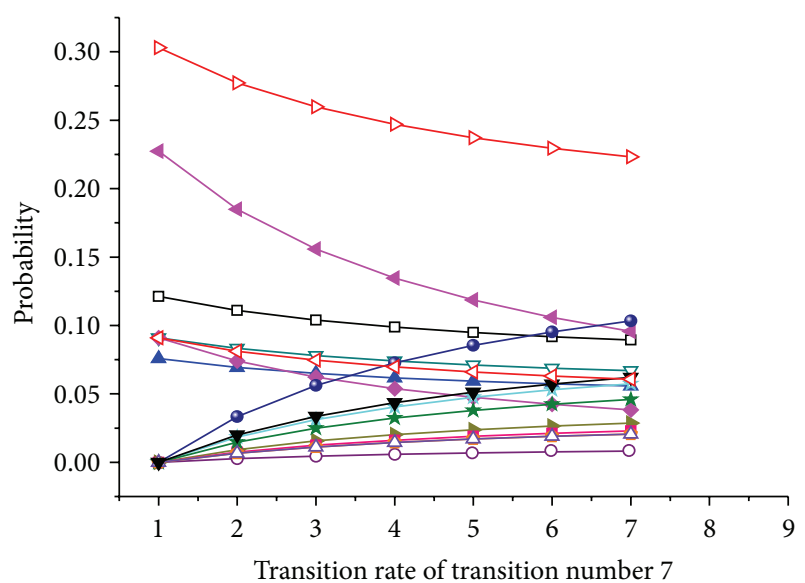

$$
\begin{aligned}
& \neg \begin{array}{llll}
p_{1} & \leftarrow p_{5} & \rightarrow p_{9} & -\circ p_{13} \\
p_{2} & \rightarrow p_{6} & \leftarrow p_{10} & \sim p_{14}
\end{array} \\
& \neg p_{3} \quad \multimap p_{7} \quad \multimap p_{11} \quad \boldsymbol{\nabla} p_{15} \\
& \neg-p_{4} \quad \rightarrow p_{8} \quad \rightarrow p_{12} \quad \triangleleft p_{16}
\end{aligned}
$$

Figure 7: Stable probabilities of varying transition rate $\lambda_{7}$.

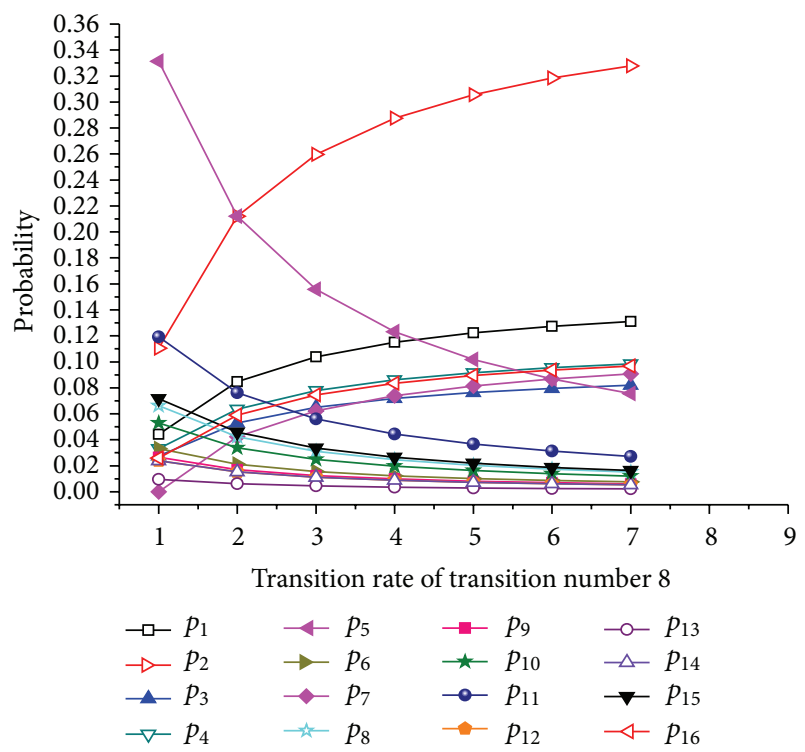

FIGURE 8: Stable probabilities of varying transition rate $\lambda_{8}$.

the probability of accidents increases evidently. The " 7.23 " and "9.27" accidents in China, which have been mentioned in the front of this paper, are both mainly caused by the ATP malfunction. The former is because of striking by lighting and the latter resulted from loss of power. Therefore, we must try our best efforts to guarantee that ATP works normally from both designing and maintaining.

From Figures 9-12, we observe that the dispatcher also plays a very important role as the second barrier for protection of train running security. Whether dispatcher can detect the abnormal timely (Figures 9 and 10) and whether dispatcher's measures work effectively after realizing abnormal situation (Figures 11 and 12) are critical to the occurrence of accident, and its influence is almost equivalent to ATP

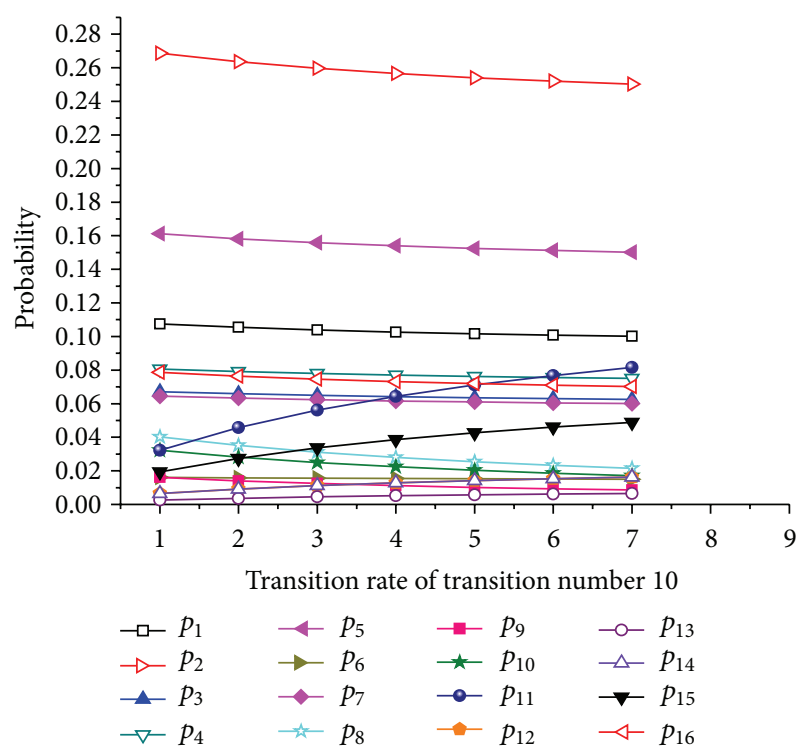

FIgURE 9: Stable probabilities of varying transition rate $\lambda_{10}$.

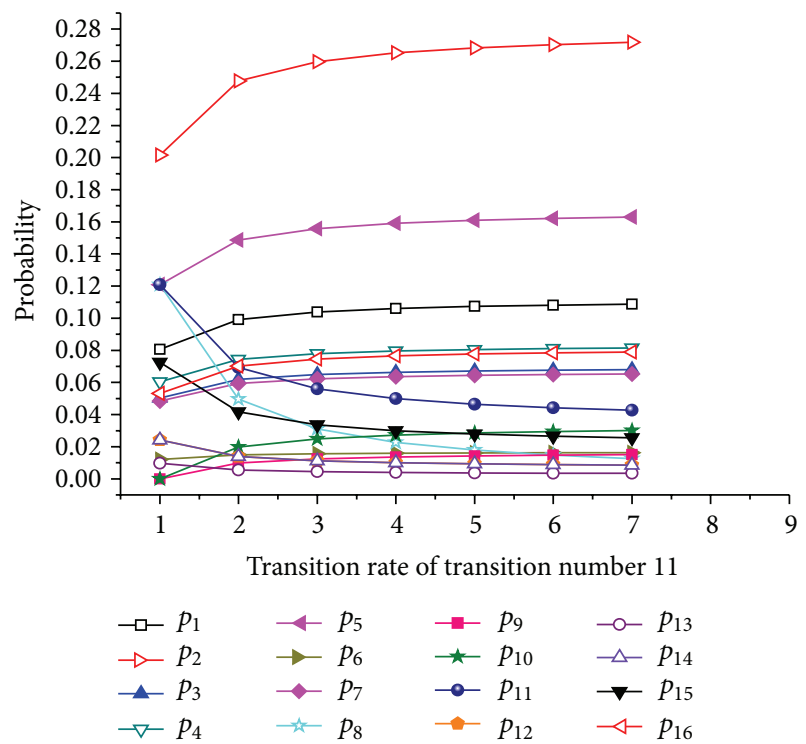

Figure 10: Stable probabilities of varying transition rate $\lambda_{11}$.

implied by the value of $P_{15}$. For instance, in the " 7.23 " accident the dispatcher is not such sensitive to the abnormal "red band" from the signal system and had not taken effective measures in the critical period of $60 \mathrm{~min}$ before the accident which resulted in the accident finally.

Figures 13-15 describe that if the frequency of $t_{18}$ and $t_{20}$ increases (Figures 13 and 14) or $t_{21}$ decreases (Figure 15), the probability of accident increases, showing that whether the driver can detect the abnormal and take effective measures is related to the occurrence of the accident. However, because drivers are generally the recipients of information, the impact is not as significant as ATP and dispatcher seen from the place's busy probability value of $P_{15}$. When the driver noticed 


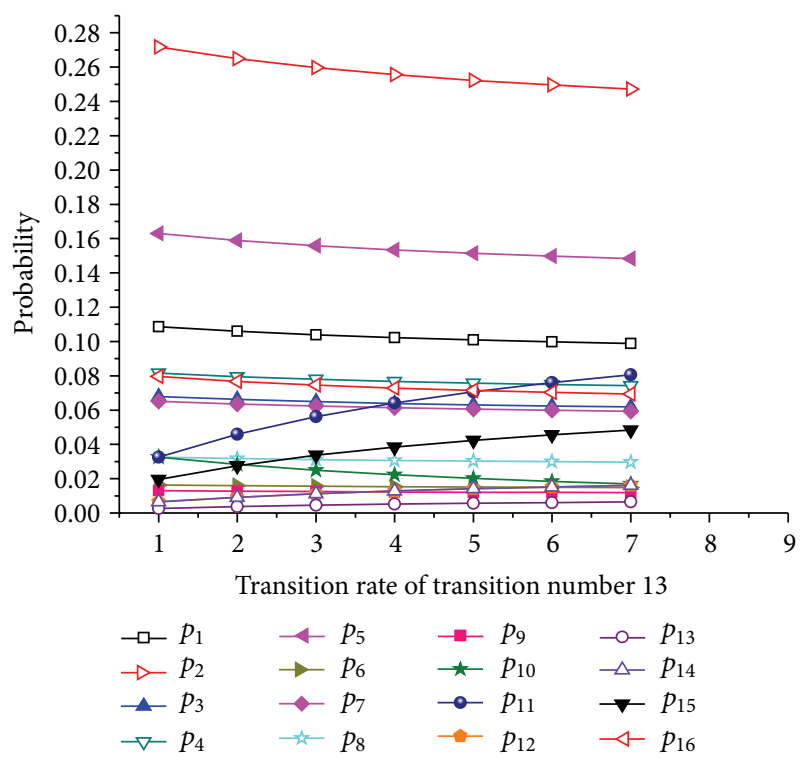

FIGURE 11: Stable probabilities of varying transition rate $\lambda_{13}$.

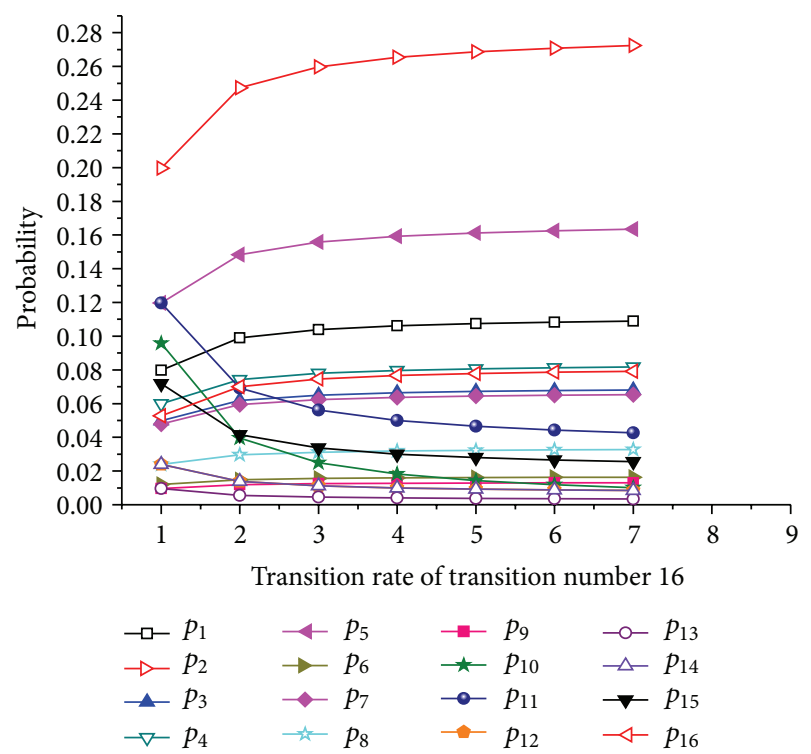

Figure 12: Stable probabilities of varying transition rate $\lambda_{16}$.

the abnormal, there was not enough time and space distance for the driver to take measures to prevent the accident.

\section{Conclusion}

In this paper, we proposed a model of train rear-end collision accidents based on the theory of SPN and verified the validity of the model based on the isomorphic MC. Meanwhile, we provided quantitative analysis of the train rear-end collision accidents by the isomorphic MC of the SPN model. In the quantitative analysis, we accounted for the uncertainties of the firing rate $\lambda_{i}$ of the transitions and introduced the triangular fuzzy numbers to fuzzy $\lambda_{i}$. We took different fuzzy degrees for $\lambda_{i}$ in the steady state probability equations of the

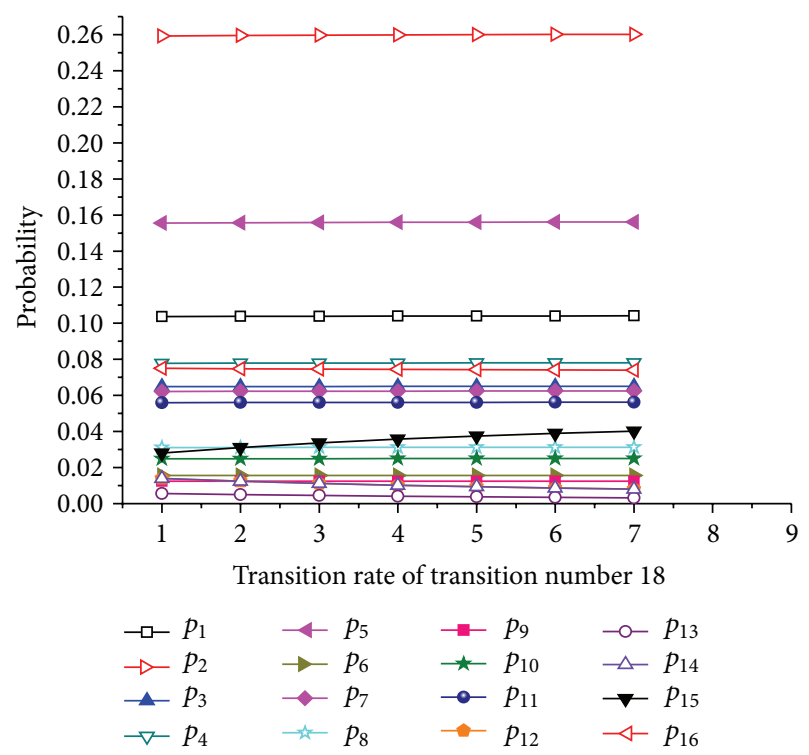

FIGURE 13: Stable probabilities of varying transition rate $\lambda_{18}$.

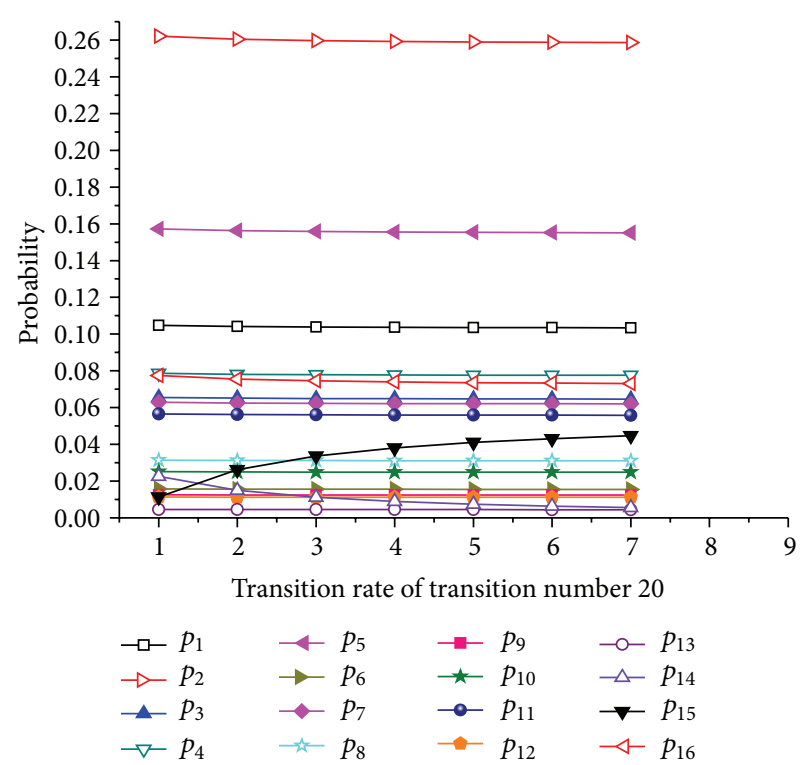

FIGURE 14: Stable probabilities of varying transition rate $\lambda_{20}$.

Markov process, converted the equations into fuzzy steady state probability equations, and solved the fuzzy equations to get the steady state probability which is more reliable.

By analyzing the steady state probability, it is found that the busy probability of places $P_{2}$ and $P_{5}$ is larger relatively. Since $P_{2}$ is the initial state of the train rear-end accident process, it is easy to reduce accidents in this state. However, it is also a risk state which is most likely to be ignored. Therefore, at the same time of reducing the possibility of generating this state, we should pay attention to timely adjustment of this state after it is generated. The busy probability of place $P_{5}$ is relatively large, indicating that the role of ATP is crucial for protection of train driving security. Meanwhile, it 


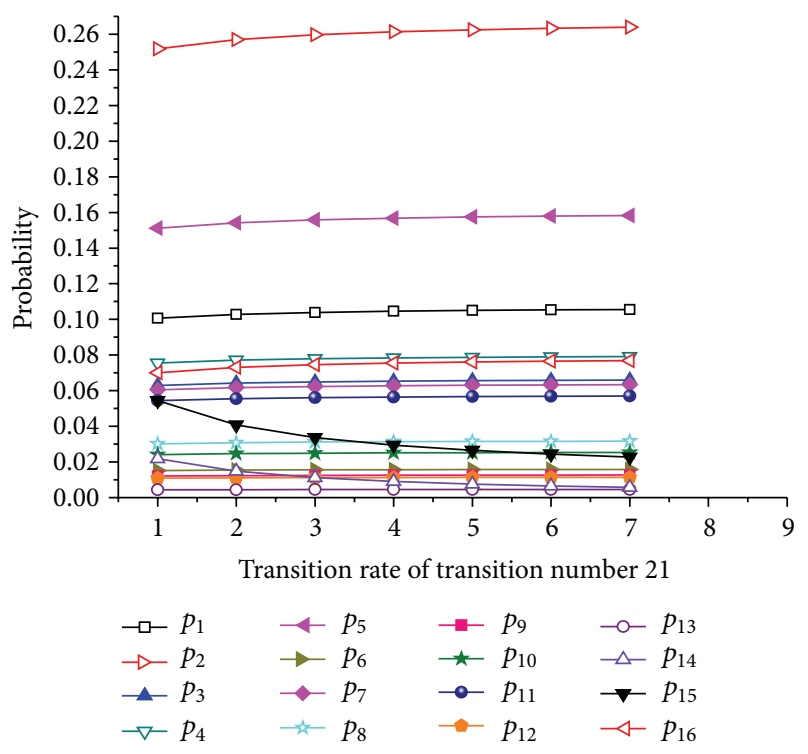

FIGURE 15: Stable probabilities of varying transition rate $\lambda_{21}$.

is consistent with the actual situation that the busy probability of the place $P_{15}$ is not large.

In all, the case analysis and discussion showed that the results of the proposed train rear-end accidents model based on SPN are reasonable in practical applications and can be used to effectively analyze the accidents or prevent loss, and the results may be useful to the department of railway safety management.

However, there are also some shortcomings in the model. In order to avoid the deadlocks, we made some idealized processing during the model design stage, such as not considering the feedback between the driver and dispatcher, ignoring the repairing and recovering of ATP after the dispatcher noticed that it is abnormal, which should be the subject of our further research.

\section{Conflict of Interests}

The authors declare that there is no conflict of interests regarding the publication of this paper.

\section{Acknowledgments}

This research was supported in part by the National Basic Research Program of China (Grant no. 2012CB725400), the National Natural Science Foundation of China (Grant nos. U1434209 and 71131001-1), and the Research Foundation of State Key Laboratory of Rail Traffic Control and Safety, Beijing Jiaotong University, China (Grant nos. RCS2014ZT19 and RCS2014ZZ001).

\section{References}

[1] A. Mirabadi and S. Sharifian, "Application of association rules in Iranian Railways (RAI) accident data analysis," Safety Science, vol. 48, no. 10, pp. 1427-1435, 2010.
[2] W. Wang, X. Jiang, S. Xia, and Q. Cao, "Incident tree model and incident tree analysis method for quantified risk assessment: an in-depth accident study in traffic operation," Safety Science, vol. 48, no. 10, pp. 1248-1262, 2010.

[3] J. De Oña, R. O. Mujalli, and F. J. Calvo, "Analysis of traffic accident injury severity on Spanish rural highways using Bayesian networks," Accident Analysis and Prevention, vol. 43, no. 1, pp. 402-411, 2011.

[4] T. Kontogiannis, V. Leopoulos, and N. Marmaras, "A comparison of accident analysis techniques for safety-critical manmachine systems," International Journal of Industrial Ergonomics, vol. 25, no. 4, pp. 327-347, 2000.

[5] N. G. Leveson and J. L. Stolzy, "Safety analysis using Petri nets," IEEE Transactions on Software Engineering, vol. 133, no. 3, pp. 386-397, 1987.

[6] Z. Z. Li, "Fault tree analysis of train rear-end accidents and a talking on the complex system security," Industrial Engineering and Management, vol. 16, no. 4, pp. 1-8, 2011 (Chinese).

[7] L. Harms-Ringdahl, "Relationships between accident investigations, risk analysis, and safety management," Journal of Hazardous Materials, vol. 111, no. 1-3, pp. 13-19, 2004.

[8] D. Vernez, D. Buchs, and G. Pierrehumbert, "Perspectives in the use of coloured Petri nets for risk analysis and accident modelling," Safety Science, vol. 41, no. 5, pp. 445-463, 2003.

[9] C. A. Petri, Kommunikation Mit Automaten [Ph.D. thesis], Shriften des IIM Nr. 2, Institute fur Instrumentelle Mathematik, Bonn, Germany, 1962.

[10] R. David and H. Alla, "Petri nets for modeling of dynamic systems: a survey," Automatica, vol. 30, no. 2, pp. 175-202, 1994.

[11] T. Murata, "Petri nets: properties, analysis and applications," Proceedings of the IEEE, vol. 77, no. 4, pp. 541-580, 1989.

[12] C. Lin, Stochastic Petri Nets and System Performance Evaluation, Tsinghua University Press, Beijing, China, 2005, (Chinese).

[13] J. Wang, Y. Deng, and C. Jin, "Performance analysis of traffic control systems based upon stochastic timed Petri net models," International Journal of Software Engineering and Knowledge Engineering, vol. 10, no. 6, pp. 735-757, 2000.

[14] M. K. Molloy, On the integration of delay and throughput measures in distributed processing models [Ph.D. thesis], University of California, Los Angeles, Calif, USA, 1981.

[15] L. A. Zadeh, "Fuzzy sets," Information and Computation, vol. 8, pp. 338-353, 1965.

[16] T. J. Ross, Fuzzy Logic with Engineering Applications, Wiley, Chichester, UK, 2009. 


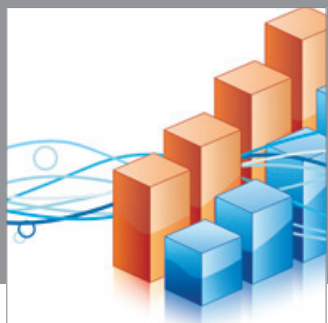

Advances in

Operations Research

mansans

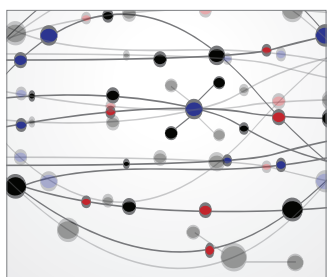

The Scientific World Journal
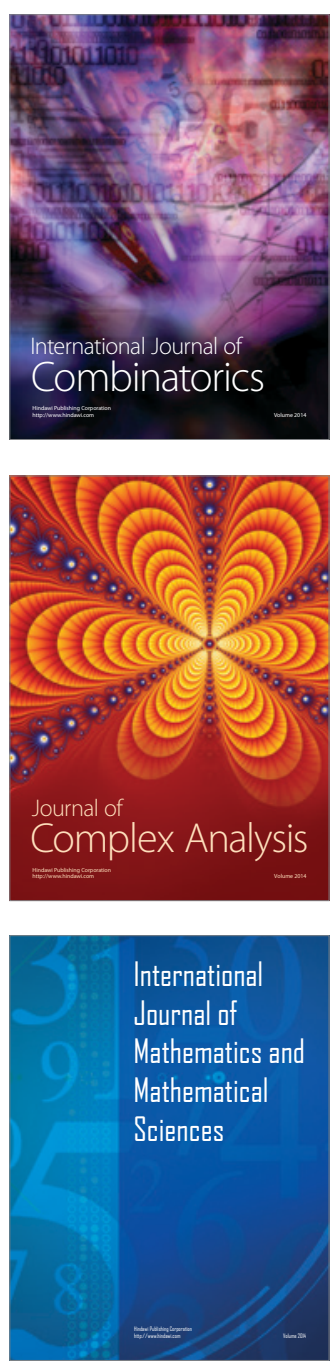
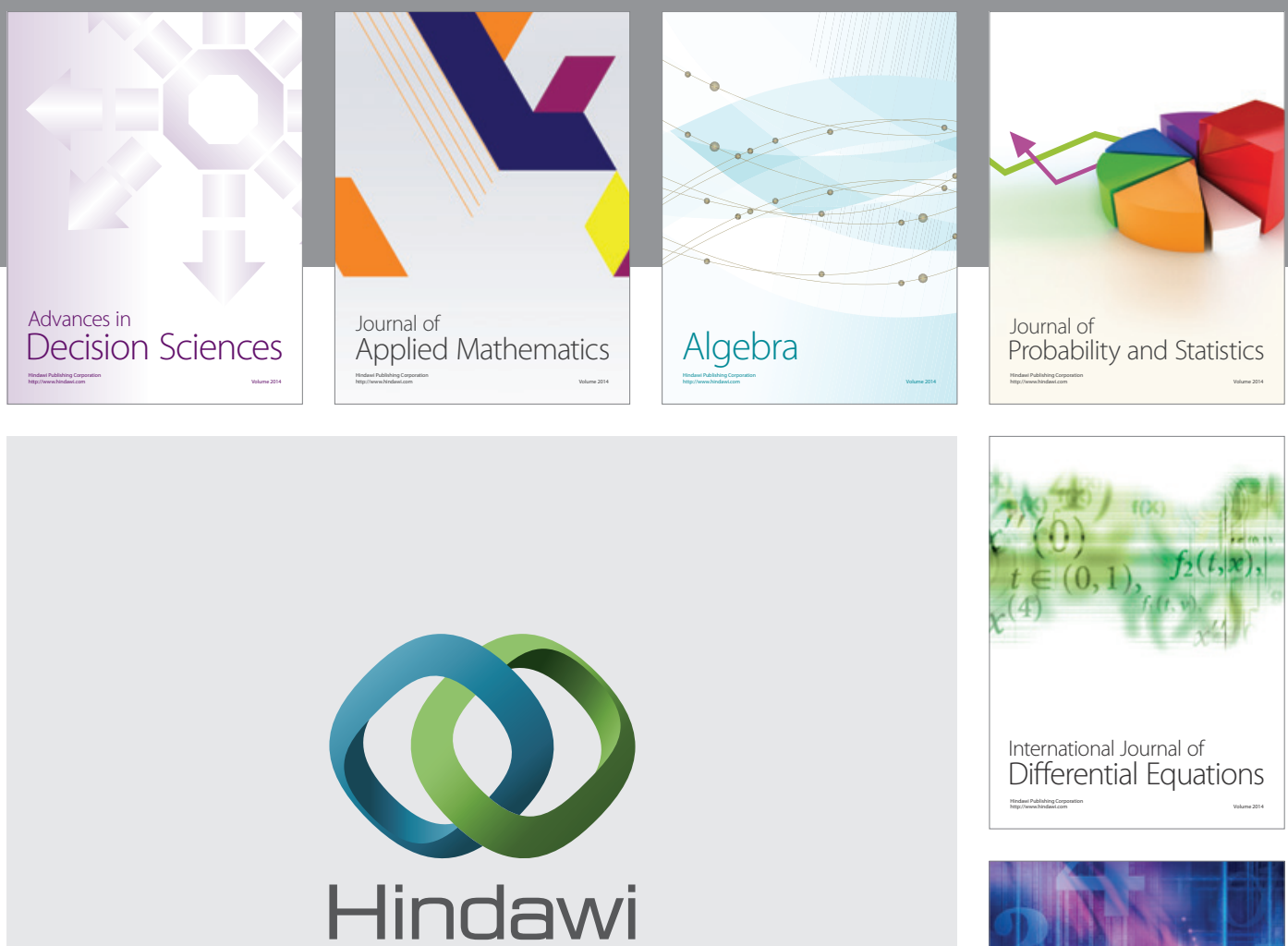

Submit your manuscripts at http://www.hindawi.com
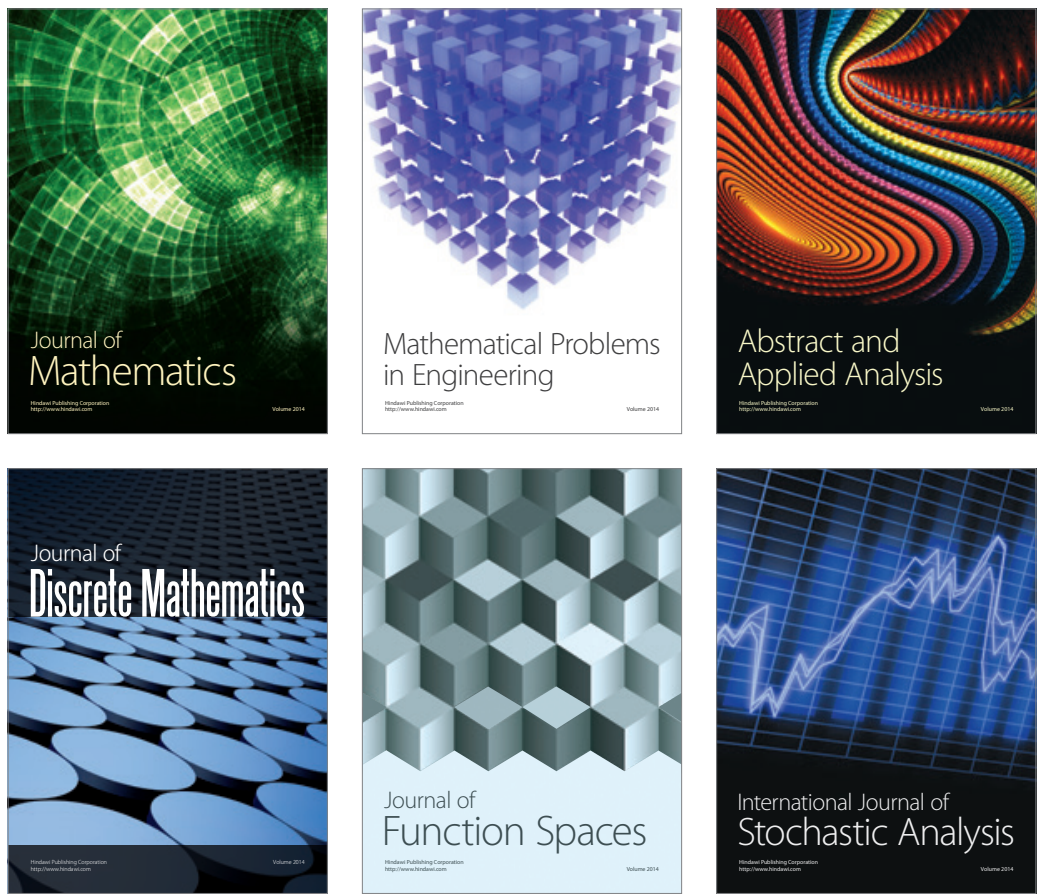

Journal of

Function Spaces

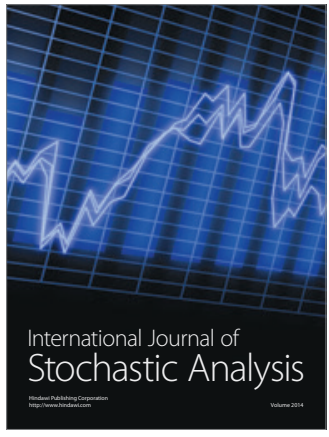

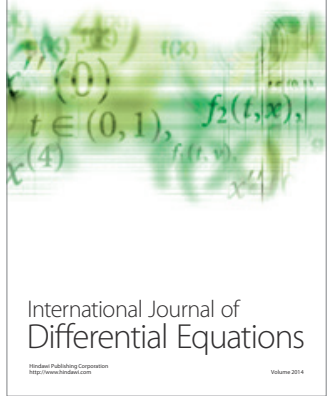
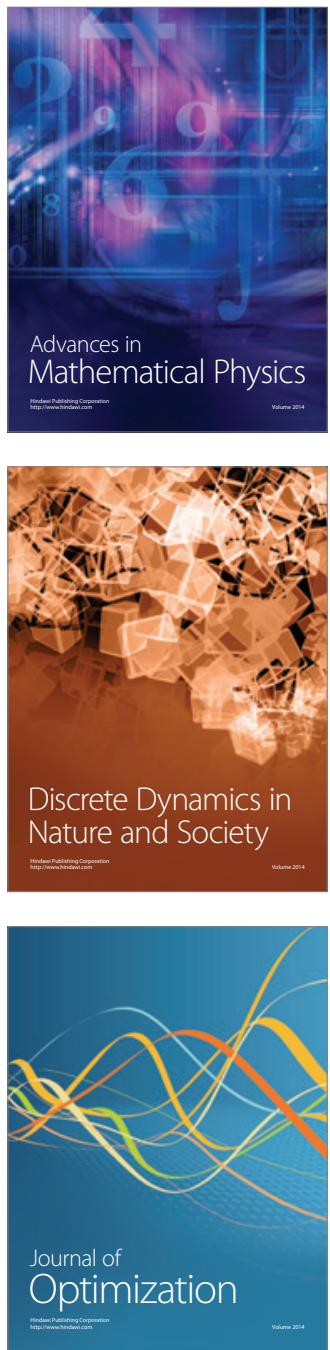